Kriging models for aero-elastic simulations and reliability analysis of offshore wind turbine support structures
A. Moratóa ${ }^{\text {* }}$, S. Sriramula ${ }^{\mathrm{a}}$, N. Krishnan ${ }^{\mathrm{b}}$
${ }^{a}$ LRF Centre for Safety and Reliability Engineering, Aberdeen, United Kingdom; ${ }^{b}$ Lloyd's
Register EMEA, Aberdeen, United Kingdom
*Corresponding author: Alexandre Morató
Lloyd's Register Foundation (LRF) Centre for Safety \& Reliability Engineering,
School of Engineering, University of Aberdeen
Aberdeen, AB24 3UE, UK.
Phone: +44 (0)1224 272000
Email: r07am14@abdn.ac.uk 


\title{
Kriging models for aero-elastic simulations and reliability analysis of offshore wind turbine support structures
}

\begin{abstract}
The existence of uncertainties in material properties, environmental loads and soil properties as well as the presence of nonlinearities introduced by the control systems have a remarkable influence on the dynamic response of offshore wind turbine (OWT) support structures. The reliability computations of these structures need to consider implicit expensive-to-evaluate limit state functions, implying large computational costs. This paper addresses these limitations by proposing a computationally-efficient reliability framework for OWT support structures, based on the use of a kriging model to approximate the response of the system, capturing both the dynamic behaviour of the structure and inherent uncertainties. The surrogate model is built with sample points from stochastic fully coupled simulations in the time-domain. A thorough sensitivity study is performed on the influence of number of sample points, the seeds used to obtain each point, the range of the variables and the inherent variability in turbulent wind and stochastic waves. The framework is used to evaluate the reliability of the NREL 5 MW turbine model, mounted on a monopile with a flexible foundation for the severest Design Load Cases (DLCs) from the IEC 61400-3. The results agreed with the general literature showing that the structure is far from failure.
\end{abstract}

Keywords: Offshore wind turbine; Design load case; Support structure; Kriging; reliability; surrogate models

\section{Introduction}

The Offshore Wind Turbine (OWT) industry is rapidly maturing with an exponential increase in the installed capacity of offshore wind farms. Nowadays, the market is moving to floating OWT concepts while the solutions for fixed structures in shallow waters are not yet optimised. Also, it has been recommended that an appropriate approach be developed to determine the target reliability levels for fixed structures in order to capture the design changes with more rational safety factors (Jha et al. 2009).

The Levelised Cost Of Energy (LCOE) is an indicator to compare the cost of energy, and it is key for the development of offshore wind to lower it through optimisation. To 
achieve that, a deeper understanding of the reliability levels delivered by the current standards is essential. The current design of OWT support structures is performed largely following the IEC 61400-3 standard (IEC 2009) based on the Load and Resistance Factor Design (LRFD), which defines several design situations representing the various modes of operation of the turbine, each deriving into a large number of Design Load Cases (DLCs), with their associated load factors. The design standard offers guidance on methods to evaluate the DLCs in order to check the structural integrity of the OWT. It was created by combining the offshore Oil \& Gas standards and the existing onshore wind energy design standards, which were first addressed in the RECOFF (Norton and Quarton 2003) project, proposing a series of recommendations for the design of OWT (Norton and Quarton 2003), and leading to the elaboration of IEC 61400-3 (IEC 2009). In spite of these efforts, it is generally acknowledged that the reliability levels achieved by following the standards are not yet clear and the resulting partial safety factors might not be optimal for OWT.

An essential step towards the understanding of the effectiveness and rationality of the partial safety factors is to develop efficient frameworks or methods to assess the reliability of such structures, and this is a challenging task due to the computational simulations involved. When time-domain simulations are employed, each evaluation of the Limit State Function (LSF) may imply a large computational cost. As a consequence, some authors limited the scope of analysis. Wei et al. (2014) used an incremental wind-wave analysis under extreme loading, using a static pushover analysis (similar to the approach used for Oil \& Gas structures), and applied it to a jacket and a monopile structure. A single and two-parameter approach was used, both leading to a very high reliability index for the monopile with a LSF based on the plastic moment; their study, however, did not account for the dynamic effects of the structure itself nor for the nonlinearities introduced by the turbine controllers. (Kim and Lee 2015a) avoided the expensive computational time domain simulations by estimating a 
dynamic peak response using static response and a dynamic amplification factor. This was applied to a jacket support structure during a parked situation under extreme loading and the LSF was linked to the displacement of the structure at mudline. A different approach was taken by Muskulus and Schafhirt (2015), by creating a decoupled linear numerical model and obtaining the structural response through impulse-response functions for an arbitrary load time series. They applied it to a monopile and jacket structure with similar results to the previous studies; the design was far from failure and the highest values were obtained for operational DLCs. Assuming that the stochasticity of the variables, the nonlinearities introduced by the turbine controls and the dynamic time domain simulations are unavoidable, (Carswell et al. 2014a) analysed the reliability of a monopile substructure using fully coupled simulations. They used the p-y method to model the soil-structure interaction and the LSFs were taken as the displacement and rotation of the pile head at mudline. It was observed that the uncertainty in soil properties greatly influences the overall reliability, while the tower damage and the associated stiffness reduction dramatically alter the tower response. Recently, Yang et al. (2015) proposed a methodology for the design optimization based on Kriging model which replaces the original decoupled numerical simulation of the Finite Element $(\mathrm{FE})$ model. They used the Von Mises stress, tower top displacement and natural frequency to optimise the structure weight and study a jacket support structure. It is however still missing a fully coupled model with aero-hydro-servo-elasto simulations which are able to comprehensively capture the most important failure modes of these structures at different levels, from tower top to the base of the foundation.

Motivated by the need to develop a computationally efficient framework for the reliability analysis and to take the applicability of the kriging approach further, in this paper we extend a methodology introduced in Morató et al. (Sept 2015) making use of stochastic fully coupled simulations, which capture both the dynamic behaviour of the structure and 
inherent uncertainties, followed by detailed finite element analysis for reliability computations. The accuracy of the different methods for constructing the kriging model is also studied. This paper also intends to gather the most common LSFs found in the literature representing common failure modes of different parts of the structure, such as the foundation, blades and the tower. The method is based on an interface between FAST 8 (J. M. Jonkman and Buhl 2005), an aero-hydro-servo-elastic simulator developed by National Renewable Energy Laboratory (NREL) and the FE analysis software Abaqus®. The Kriging method is proposed to approximate the outcomes of the computational interface. First, several design points are generated through a random sampling technique. Then, using these points, a model is constructed to replace the original computational interface. Finally, Monte Carlo simulations, First and Second Order Reliability methods (FORM and SORM) are used to perform the reliability analysis and derive the reliability index. By using a surrogate model, additional stochastic variables such as material and geometric properties, as well as loads can be incorporated in the analysis. Also, the Apparent Fixity (AF) (Bush and Manuel 2009) method is used to model a flexible soil-pile interaction, thus additional uncertainties coming from the soil properties are also incorporated. The approach is demonstrated by applying to the benchmark NREL 5 MW monopile OWT (J. M. Jonkman et al. 2009).

\section{Kriging models}

\subsection{Metamodels and reliability}

The most widely used and established method for the reliability analysis is the Monte Carlo simulation (MCS) method. This technique is probably the best option for reliability problems with explicit limit state functions which are "easy" or "cheap" to evaluate. It can also be improved with variance reduction techniques such as importance sampling or subset simulations when higher accuracy is needed around the failure region. However, when it comes to complex structural systems, powerful and advanced numerical models might be 
required for the reliability analysis. Besides, OWT structures are normally designed to obtain very low probabilities of failure, which implies that an implicit LSF be solved several thousand times, resulting in a prohibitively large computational effort. A solution for this problem is to use metamodels, also known as surrogate models or response surface methods. A metamodel is nothing but a model of a model: it constructs a relationship between the design variables and corresponding responses using relatively few sampling points and the original complex model is effectively replaced by a transfer function called response surface. The LSF is no longer an implicit expensive-to-evaluate function but an explicit function, which can now be used with traditional reliability methods. There are different types of metamodels, of which the polynomial response surfaces method is the most common one used in engineering problems. It was first applied to a structural problem by (Bucher and Bourgund 1990) in which a two-stage method was applied with a quadratic response surface function. Kriging method is another type of surrogate model that uses interpolation based on the assumption that there is a spatial correlation between the model predictions (Kaymaz 2005). Kriging was originally developed in geostatistics (also known as spatial statistics) by the South African mining engineer called Krige. The Kriging models do not assume an underlying global functional form as assumed in the polynomial regression models (e.g. firstor second-order polynomials) and can approximate arbitrary functions with high accuracy in global as well as local levels. These models are frequently described in the literature as the realisation of a stochastic field or Gaussian processes. Kaymaz (Kaymaz 2005) compared the Kriging method with the polynomial response surface method using different numerical examples, including the effect of the kriging parameters, and observed that the kriging method showed great accuracy. Also, several researchers proposed different correlation functions and studied the compatibility with the regression functions in different industries (Rasmussen 2006; Santner, Williams, Notz 2013). In recent years, the potential of the 
Kriging method for structural reliability estimates has been discovered, resulting in many relevant studies assessing its efficiency (Gaspar et al. 2014; Shi et al. 2015; Zhang et al. 2015a).

\subsection{Fundamentals of Kriging models}

The idea of Kriging method is to estimate the value of the output from the sum of the weighted values of the known surrounding sample points $X=\left(x_{1} \ldots x_{n}\right)$ with $x_{i} \in \mathbb{R}^{m}$. The corresponding responses $Y=\left(y_{1} \ldots y_{n}\right)$ with $y_{i} \in \mathbb{R}^{p}$ of these experimental points $x_{i}$ are obtained from the complex numerical model simulations. Then, a predicted value $\hat{Y}$ is expressed in two parts as

$$
\hat{Y}(x)=f(x)+\delta(x)
$$

where $f(x)$ is the regression function (or trend) and is based on the data, and the Gaussian

process $\delta(x)$ is constructed through the residuals with mean 0 , variance $\sigma^{2}$ and a covariance

$$
\operatorname{Cov}\left(x_{i}, x_{j}\right)=\sigma_{l}^{2} R\left(\theta, x_{i}, x_{j}\right), \quad l=1, \ldots, p
$$

where $R\left(x_{i}, x_{j}\right)$ is the correlation function, $\sigma_{l}$ is the process variance for the $l^{\text {th }}$ component and $\theta$ represents the hyperparameters. The regression function is defined as a multivariate polynomial which takes the form

$$
f(x)=\sum_{i=1}^{n} \beta_{i} . b_{i}(x)
$$

where $\beta_{i}$ denotes the coefficients and $b_{i}(x)$ denotes the basis functions such as the power base for a polynomial. In some kriging variants, the regression function might be taken as 0 
(e.g. simple kriging) or set to a constant $f(x)=b_{0}$ (e.g. ordinary kriging). In simple words, the regression function captures the general trend of the data and the Gaussian Process interpolates the residuals. Selecting the correct regression function is a difficult problem; hence, the regression function is often chosen as a constant, which has been proven to be enough for common engineering problems.

The stochastic part shown in Equation (1) includes the correlation function, which is a function of the distance between surrounding data point and the distance between data points and the predictor point, respectively. The correlation functions are semivariograms that define the degree of spatial correlation between data points in stochastic processes, a correlation function of the type $R\left(\boldsymbol{x}_{\boldsymbol{i}}, \boldsymbol{x}_{\boldsymbol{j}}\right)=R\left(\boldsymbol{x}_{\boldsymbol{i}}-\boldsymbol{x}_{\boldsymbol{j}}\right)$, is generally selected (Sacks et al. 1989), which is largely dependent on the separation distances. The correlation function $R\left(\boldsymbol{x}_{\boldsymbol{i}}, \boldsymbol{x}_{\boldsymbol{j}}\right)$ is to be chosen and several correlation functions are suggested in the literature. The Gaussian and exponential correlation functions are probably the most used, however, others such as the simplified Matérn functions with shape parameter $v=3 / 2$ and $v=5 / 2$, known as Matérn-3/2 and Matérn-5/2 respectively, are also suggested in the literature. Others less common could be the linear, spherical or circular correlation functions. For example, the correlation function for the distance between a data point $x$ and the predictor point $x_{0}$ will take the form:

- Gauss / exponential

$$
R\left(x-x_{0}, \theta, q\right)=\exp \left(-\frac{\left|x-x_{0}\right|^{q}}{\theta}\right)
$$

- Matérn 


$$
R\left(x-x_{0}, \theta, v\right)=\frac{1}{2^{v-1} \Gamma(v)}\left(2 \sqrt{v} \frac{\left|x-x_{0}\right|}{\theta}\right)^{v} \mathrm{~K}_{v}\left(2 \sqrt{v} \frac{\left|x-x_{0}\right|}{\theta}\right)
$$

where $q$ denotes the smoothness of the function; the function is either Gaussian or exponential when $q=2$ or $q=1$, respectively, $\Gamma$ is the Euler Gamma function, $K_{v}$ is the Bessel function of the third kind and $\theta \in \mathbb{R}^{m}$ denotes the hyperparameters of the function, which are calibrated using the Maximum Likelihood Estimation (MLE).The dimension of the hyperparameter is the same as the dimension of each sample point, which is the number of random variables. It is interesting to note that an unreliable prediction will be derived if there are large distances or few clusters surrounding the predictor location. On the other hand, kriging is the best linear unbiased estimator for intermediate points. Also, a data point will have different kriging weights for another predicted estimation. Figure 1 show how the correlation function may differ from each other depending on $\theta$ and how each function can change as a function of $\theta$ as well.

\section{Reliability analysis approach}

\subsection{Fully coupled simulations}

The dynamic analysis is performed using an interface between an aero-hydro-servo-elastic simulation and structural finite element analysis, both linked with Matlab®, which allows the simulations to run in batch mode. All the hydrodynamic, aerodynamic and internal nonlinear loading are calculated using FAST v8 (developed by Jonkman and Buhl at NREL (J. M. Jonkman and Buhl 2005)), and Abaqus ${ }^{\circledR}$ is chosen to perform the finite element structural analysis. The interface between FAST and Abaqus enhances the opportunities for efficient reliability computations, capturing the possible nonlinearities as the analysis progresses. However, in this paper the interface was only used for Von Mises stress. Also, both material and geometry nonlinearities are introduced in the reliability analysis through variables. 
FAST v8 uses a modularisation framework to simulate the non-linear coupled dynamic performance of offshore and onshore Horizontal-Axis Wind Turbines. The aerohydro-servo-elastic tool FAST is used as an interface between different modules such as AeroDyn, HydroDyn, ServoDyn, ElastoDyn and SubDyn that feed it. The wind acting on the rotor and tower, the global structural dynamics and the hydrodynamic loading are reduced to tower top resultant loads (3 forces and 3 moments) and a hydrodynamic moment at the seabed. These loads calculated by FAST are then applied to the Abaqus ${ }^{\circledR}$ model, which performs a time-domain dynamic analysis applying the time-varying tower top loads and hydrodynamic moment. The introduced material and geometric uncertainties capture the influence of parameters such as thicknesses or Young's modulus. A scheme representing the interface can be seen in Figure 2 and further information about this computational framework and its validation can be found in (Morató et al. 2015).

The turbulent wind field is computed by TurbSim (B. J. Jonkman 2009). The conventional method for controlling power-production operation relies on the design of two basic control systems: a generator-torque controller and a full-span rotor-collective bladepitch controller. The goal of the generator-torque controller is to maximize power capture below the rated operation point. On the other hand, blade-pitch controller aims to regulate the generator speed above the rated operation point by feathering (pitching) the blades, limiting the thrust force and keeping the outputted power constant. ServoDyn calls the already-proven and validated NREL offshore 5-MW wind turbine's baseline control system, which was developed by NREL as an external Dynamic Link Library (DLL). Further information about this routine can be found in (J. M. Jonkman et al. 2009). It is assumed that the wind turbine is class II within the framework found in IEC (IEC 2005). The turbulence reference intensity is chosen as B (0.14) as class $\mathrm{A}$ is unlikely to be found offshore, unless the spacing within the wind farm is lower than typically found, and hence quite conservative. HydroDyn uses 
Morison's equation to model the hydrodynamic loading. Here, depending on the sea state condition, either Airy's or $2^{\text {nd }}$ order stokes theory are used to derive wave kinematics and then evaluate the inertia and drag loading (through an inertia coefficient and a drag coefficient). The monopile is treated as a slender Morison's element and thus the use of potential theory (radiation and diffraction) is not needed. The current is modelled as a nearsurface current: the model follows a linear relationship down to a reference depth. Further information can be found in FAST user guide (J. M. Jonkman and Buhl 2005).

\subsection{Limit state Functions}

There is a significant amount of literature focusing on the reliability of OWT structures (Agarwal 2008; Carswell et al. 2014; Kim and Lee 2015; Muskulus and Schafhirt 2015), however most of it deals with substructures, structures or blades separately. This paper takes advantage of the framework presented, which performs fully coupled simulations using a flexible foundation, being capable of bringing together the LSFs analysis from the tower top to the foundation level. The LSFs used herein show the main LSFs currently used in general research for this type of structures for ULS or Serviceability Limit State (SLS) such as plastic yielding, local buckling or maximum displacement or rotation at certain points of the structure.

One example of LSFs is the condition that the maximum Von Mises stress reaches yield at any point in the structure. It is used in (Yang et al. 2015) as a reliability-based design optimization constraint and in (Morató et al. 2016) for reliability evaluation. This is a very conservative condition as first yielding at a point does not necessarily imply collapse. The highest $P_{f}$ is expected to come from this equation, expressed as follows:

$$
g=f_{y}-\sigma_{V M}
$$


where $f_{y}$ is the yield strength, and $\sigma_{V M}$ denotes the maximum equivalent stress. Another ULS failure criteria of a structure is likely to come from plastic yielding; it is related to the cross section strength and it occurs when the acting bending moment is higher than the bending resistance to plastic yielding. Beyond that stress state, the material starts deforming plastically and a hinge is formed. This is assessed with a simplified failure criterion based on the design standards (DNV 2010 and NORSOK 2004) shown in Equation 6 at the relevant points on the structure, i.e., tower base and mudline level as:

$$
g=Z f_{y}-S_{M}=\frac{1}{6}\left(D^{3}-(D-2 t)^{3}\right) f_{y}
$$

where $Z$ is the plastic section modulus expressed as a function of the diameter $D$, and the thickness $t, S_{M}$ is the acting bending moment at the corresponding section. This LSF was used for reliability-based design in (Muskulus and Schafhirt 2015) and to assess the structural capacity in (Wei et al. 2014).

For OWT structures the factor $D / t$ is of great importance for cost reduction (less volume of steel) and in dynamic assessment to match the desired natural frequency. The tendency is to use as high a ratio as possible, which may cause failure under local buckling. We use a simplified model for local buckling failure of an OWT support structure in shallow waters, as explained in (Sørensen and Toft 2010; Sorensen and Tarp-Johansen 2005) for optimization purposes and also used for the reliability assessment in (Morató et al. 2016). The LSF is written as follows,

$$
g=M_{c r}-S_{M}
$$

where, $M_{c r}$ resistant bending moment, and $S_{M}$ represents the bending moment at the tower base level. The resistance $M_{c r}$ can be obtained as 


$$
M_{c r}=\frac{1}{6}\left(1-0.84 \frac{D}{t} \frac{f_{y}}{E}\right)\left(D^{3}-(D-2 t)^{3}\right) f_{y}
$$

where $D$ and $t$ are the diameter and thickness at tower base level respectively, and $E$ denotes the Young's Modulus. It applies a reduction factor to account for buckling on the plastic moment which directly depends on the magnitude of $D / t$. As seen in Figure 3, for a given diameter $(6 \mathrm{~m})$, the higher the $D / t$ the lower the buckling resistance and the bigger the difference with the plastic moment. As for the plastic yielding failure criterion, the model for local buckling is applied at the tower base and mudline level, which are critical locations for the substructure integrity. The first one has the section with highest $\mathrm{D} / \mathrm{t}$ ratio and it is where the transition piece is located, whereas the second one withstands the severest moments and interacts with the soil.

Another type of LSF for a ULS is the gap between the tower and the tip of the blade. This parameter is critical for the design of the blades as it limits their length. If this distance is 0 the blade crashes against the tower causing failure of the blade and most likely damaging the tower severely. An additional LSF relates to the foundations, typically defining a limit for excessive deflection/rotation. Some authors used this type of LSF limiting the displacement and rotation at the tower top (Yang et al. 2015) and at the mudline level if a flexible foundation is used (Carswell et al. 2014a; Carswell et al. 2014b).

\subsection{Creation of the metamodel and reliability analysis}

As explained previously, the kriging models are used to approximate responses such as the Von Mises stress $\left(\sigma_{V M}\right)$ or the bending moment at tower base level $(M)$ with the aim of obtaining the response surface; $\sigma_{V M, k r i g}$ and $M_{k r i g}$. The first step in the creation of a metamodel is to define the number of random variables (i.e. thickness, Young's modulus, mean wind speed, $H_{S}$, etc.), their distributions and the distribution parameters. Subsequently, 
the Design of Experiments (DoE) is carried out defining the number of the sample points and an efficient spatial distribution. There are different techniques for DoE such as Central Composite Design (CCD) (Barker 2005), Box-behnken (Box et al. 1978) or Latin Hypercube Sampling (LHS) (Wang 2003). In this paper, the LHS sampling technique is chosen and the number of sampling points are carefully picked. A good initial range for the stochastic variables to cover $99 \%$ of the probabilities is three times the standard deviation.

The computational interface described in Figure 4 is used to obtain the structural response (i.e. tower base bending moment, displacement at mudline, etc.) for the chosen sample points, and the outputs vector $(p=1)$ corresponding to the sample points matrix are formed (i.e. maximum tower base bending moment of each simulation). In order to apply the kriging method, two specific Matlab® toolboxes available in the public domain called are used, see Section 5. The kriging result is a very long expression which is not tractable manually but computationally-cheap to evaluate. A cross-validation plot may be used to check the accuracy of the model. It is interesting to note that the kriging method is the best linear unbiased predictor for intermediate points and hence an almost perfect fitting at the sample points is expected. The last part of Figure 4, shows the reliability technique used for the analysis. In this case MCS and FORM/SORM (Hohenbichler et al. 1987) are used to derive the probability of failure of each LSF for each DLC.

\section{APPLICATION EXAMPLE}

\subsection{Benchmark and site specifics}

The structure used for the study is the 5MW monopile OWT model from the OC 3 project with a water depth of 20m (J. Jonkman and Musial 2010). The platform has a constant thickness of $0.06 \mathrm{~m}$ with a diameter of $6 \mathrm{~m}$ whereas the tower diameter and thickness decrease linearly, the diameter from 6 to $3.87 \mathrm{~m}$ and thickness from 0.027 to $0.019 \mathrm{~m}$, further information can be found in (J. M. Jonkman et al. 2009). The location chosen for this study is 
the Ijmuiden Shallow Water Site from the Upwind design basis (Fischer et al. 2010). The site is found in the Dutch North Sea, with coordinates $52^{\circ} 33^{\prime} 00^{\prime \prime}$ north and $4^{\circ} 03^{\prime} 30^{\prime \prime}$ east.

\subsection{Flexible foundation model}

The difference between using a rigid or flexible foundation in the dynamic analysis of an OWT may significantly influence the structural response as shown in (Bush and Manuel 2009). In this paper, the flexible apparent fixity (AF) model is considered, as it is the only way to model a flexible foundation in FAST. The idea of the AF model is to replace the interaction between the pile and the surrounding soil by a fictitious cylinder that is fixed not at the original mudline but at a lower depth, as in Figure 5. The depth of the cantilevered beam and its flexural rigidity are derived so as to have the same stiffness as the true pile-soil system.

It is important to highlight that the properties of this fictive cylinder are totally dependent on the loading conditions; each different cylinder will correspond to specified levels of shear and moment at the true mudline. To determine the depth and flexural rigidity of the fictitious cylinder this procedure is followed: first, a stochastic simulation with the desired wind and wave conditions is carried out using a fixed-based model and the timeseries of shear and moments at mudline are obtained. Second, the true pile with the true soil layers and conditions is modelled in a pile lateral load analysis program such as OPILE®. Then, 50 random pairs of shear and moment from the fixed-base simulation are picked and applied in OPILE® at the pile head in order to obtain 50 pairs of pile head displacement and rotation. The length $(L)$ of the fictive cylinder and the flexural rigidity $(E I)$ are chosen such that they will produce the same rotation $(\theta)$ and lateral deflection $(w)$ at its free end (original mudline) under the shear $F$ and moment $M$ applied at the mudline. Then the parameters $L$ and $E I$ for each pair of $F$ and $M$ are derived using the following equations: 


$$
\begin{gathered}
w=\frac{F L^{3}}{3 E I}+\frac{M L^{2}}{2 E I} \\
\theta=\frac{F L^{2}}{2 E I}+\frac{M L}{E I}
\end{gathered}
$$

The apparent fixity length and the flexural rigidity values used in the AF model for the given sea state are taken as an average of the 50 pairs of $w$ and $\theta$. The results for the considered DLCs are shown in Table 1.

\subsection{Design load cases}

The aim of this study is to analyse the reliability of the well-known 5MW NREL baseline OWT benchmark structure for the severest DLCs from the IEC (IEC 2009). It is worth to mention that this structure was not designed following the IEC, but rather specified based on industry trends and information from other reference turbines. Consequently, the resulting reliability is not the one delivered by the standards. Therefore, the results shown herein ought not to be used in reliability-based code calibration, but as an indicator of the reliability of the structure. The benchmark structure is explained in more detail in the following sections.

The selection of the DLCs is based on a previous study (Morató et al. 2017), which systematically analysed a subset of Ultimate Limit State (ULS) load cases proposed by the IEC 61400-3, identifying the most critical among them in terms of key design parameters. The results showed that the highest overturning moment at the mudline level was caused by DLC 1.6a and 6.2a, both driven by the hydrodynamic loading. For this study we picked the first 3 stochastic DLCs from the ranking, which are DLC 1.3, 1.6a and 6.2a. Reliability techniques are applied to the DLCs with specific conditions for each DLC, which are explained in the following subsections. 


\subsubsection{DLC 1.3}

This power production DLC uses the Extreme Turbulent Model (ETM), Normal Sea State (NSS) with the significant wave height $\left(H_{S}\right)$ conditioned on the wind speed, with the wave kinematics based on linear wave theory, Normal Current Model (NCM) and the Mean Sea Level (MSL) during operational conditions. It is simulated with a slight misalignment to account for the lack or delay of yaw controller. The DLC requires six 10-minute simulations for each mean wind speed. The most demanding conditions within the DLC appeared for an $8^{\circ}$ yaw angle and a wind speed close to rated $(14 \mathrm{~m} / \mathrm{s})$ which corresponds to a $H_{s}$ of $1.91 \mathrm{~m}$ and a peak spectral period $\left(T_{p}\right)$ of $6.07 \mathrm{~s}$.

\subsubsection{DLC 1.6a}

The DLC 1.6a requires the Normal Turbulent Model (NTM) and follows the Severe Sea State (SSS) with a significant wave height conditioned to the wind speed $H_{S}=H_{S, S S S}(V)$. The standard allows using the unconditional extreme significant wave height $H_{S 50}$ with a recurrence period of 50 years as a conservative value for $H_{s, S S S}(V)$ (IEC 2009). The analysis uses $H_{S 50}$, implying that $H_{S}$ stays constant for all wind bins. The $H_{S}$ for a 1-hour simulation period may be obtained from the value corresponding to a 3-hour reference period (IEC 2009) using a conversion factor of 1.09. The current model is NCM, and the water offset is taken as the highest value within the Normal Water Level Range (NWLR), which is the Highest Astronomical Tide (HAT). In that case, the most demanding situation came with $8^{\circ}$ of yaw angle and the rated wind speed $12 \mathrm{~m} / \mathrm{s}$, with the value of $H_{S 50}$ taken as 8.07 and $T_{p}$ as 11.3s (Morató et al. 2017). In this case, giving the combination of $H_{s}, T_{p}$ and water depth, the Airy wave assumption is not reliable anymore: the appropriate wave theory would be stokes 3rd order. However, HydroDyn only supports formulations up to stokes 2nd order, which is used instead. This applies to section 4.3.3 as well. 


\subsubsection{DLC 6.2a}

DLC 6.2a recreates a parked/idling condition, which aims to simulate a special event: the loss of network of the wind turbine, which means that if the turbine does not have a battery backup of the yaw drive it will lose control over it. The way to model this extra condition is by running the simulations for the full range of possible yaw misalignment angles, which is $\pm 180^{\circ}$. It requires the Extreme Wind Model (EWM) with the Extreme Sea State (ESS) and wave kinematics based on second order. The DLC uses the Extreme Current Model (ECM), as well as the Highest Still Water Level (HSWL) within the Extreme Water Level Range (EWLR). It was found in (Morató et al. 2017) that the combination leading to the highest overturning moment was with $30^{\circ}$ of wind/wave misalignment and a $90^{\circ}$ and $-90^{\circ}$ of yaw angle. The conditions for the three DLCs are summarized in Table 2.

\section{Sensitivity analysis}

The effectivity of the metamodels have been already proven satisfactory for reliability systems where the outputs of the experiments follow an expected trend (Kaymaz 2005; Gaspar et al. 2014; Zhang et al. 2015) . However, the effectivity of this methodology when the transfer function is found for noisy data or for points which are outputs from aero-elastic simulations is still unpredictable. Time-series of coupled simulations include strong nonlinearities due to the control systems, and the intrinsic randomness due to turbulent wind and/or stochastic wave profiles. This section aims to study the sensitivity of the kriging model applied to aero-elastic simulations for the design of offshore wind turbines. In the following subsections, different sensitivities are analysed, such as the effect of the implicit randomness in the wind turbulence and water surface, the range of the variables, the number of experimental points and seeds as well as the kriging functions themselves. To do that, a large number of simulations is carried out with different combinations of steady/turbulent 
wind, regular/irregular waves, number of sample points and seeds used to construct each sample point.

One of the advantages of using the Kriging model to estimate the response of an aeroelastic simulation is that each simulation can output any requested output channel so, if necessary, the channel to be modelled can also be chosen after the simulations are carried out, which adds flexibility to the proposed framework for reliability. It is also important to mention that some extra variability may be expected as the random variables used for the sample points such as the mean wind speed or $H_{S}$ are used to create frequency spectra, which then results in a random turbulent wind field and random wave profile, producing different time-series (with same variance) for the same mean values. To validate the overall behaviour of each model, the Root Mean Squared Error (RMSE) is taken as an excellent generalpurpose error metric for numerical predictions, as it amplifies and severely punishes large errors:

$$
R M S E=\sqrt{\frac{1}{n} \sum_{i=1}^{n}(\widehat{Y}-Y)^{2}}
$$

where $\mathrm{n}$ is the number of validation points, $\hat{Y}$ and $Y$ are the predicted and the actual values for each of them.

To construct the Kriging models, we used two Matlab® toolboxes available on the public domain. The first one, ooDACE (Couckuyt et al. 2014) is being developed at Ghent University and it is an object-oriented toolbox for building kriging surrogate models. The outcome from the ooDACE toolbox is compared with the results obtained using the framework for uncertainty quantification developed by the UQLab at the ETH in Zurich (Lataniotis et all. 2015). This framework provides the tools to compute kriging parameters or to perform reliability or sensitivity analysis. The toolbox provides highly customisable and 
advanced options for fitting and optimising the metamodeling, allowing us to compare the results using different regression/correlation functions as well as optimisation algorithms.

\subsection{Regression and correlation functions and sample points}

Firstly, a sensitivity analysis is performed on the regression and correlation functions as well as the number of sample points used to construct a Kriging model and the range of the random variables. Four sets with 25, 50, 75 and 100 points are chosen, as well as an extra set of 125 points that is used to validate each model. Then, each set of sample points is used to construct different models with different regression and correlation functions.

Two stochastic variables are chosen in this section, the mean wind speed $\left(V_{r}\right)$ and the thickness at the tower base $\left(t_{\text {tower }}\right)$, which affects the whole tower as it changes linearly. The first variable is related to the loading and the second one to resistance. In this sub-section, the range of $V_{r}$ is kept within the rated wind speed bin [10-12m/s], whereas the range of $t_{\text {tower }}$ is $[\mu-3 \sigma, \mu+3 \sigma]$. Regarding the Kriging model, the regression functions studied are simple (with 0 mean), ordinary (unknown mean), linear and quadratic, whereas the correlation functions are Gaussian, Matern 3/2, Matern 5/2, linear and exponential. The results in this section are presented for the Von Mises stress at the base of the tower for different combinations of regression/correlation functions. To make the error dimensionless, the RMSE is divided by the mean of the actual values of the validation points, also the scale is kept the same for all the subplots to better capture the differences. The results showed that kriging models approximate very well the response of the wind turbine when the inherent variability is low, such as steady wind and still water or regular waves. Then, the accuracy tends to decrease when randomness is included through irregular waves or turbulent wind. The same happens for the influence of the number of sample points: the lesser the variability in the simulations, the clearer the improvement with more sample points; whereas in cases 
with turbulent wind and/or irregular waves the effect of the number of sample points is minor. Results are shown in Figure 6 using ordinary kriging.

Regarding the influence of the regression and correlation functions, the results look better overall with a linear regression function and the Matern 5/2 correlation function. However, ordinary kriging and linear regression function seem quite consistent, delivering an RMSE around 2.5-3\% (Figure 6c) and 2-2.5\% repectively in the worst case with turbulent wind and irregular waves. We also noticed that linear and quadratic regression functions do not get along with linear and exponential correlation functions, giving incongruences when fitting the model. The kriging models using linear regression function with Matern 5/2 and ordinary kriging with exponential correlation functions are shown in Figure 7 for a model with 50 sample points, turbulent wind and stochastic irregular waves. Notice why the kriging models are the best linear unbiased predictors for interpolation points -the black dots are the predicted values of the sample points and the surface fits them perfectly-.

\subsection{Stochasticity of waves}

This section aims to understand the contribution of the different types of waves (regular and irregular) and wind profiles (steady and turbulent) to the kriging model error, as well as that of the number of sample points and the range of the variables. The mean wind speed is one of the most important variables for this type of simulations and many design load cases include simulations within the cut-in to cut-out range. It has already been seen that one of the consequences of the control system is that the structure is most loaded when the wind speed is close to rated (Morató et al. 2017) One may approach this situation in two ways: to carry out the simulations with a mean wind speed only within the rated wind speed bin $\left(V_{r}=\right.$ $\pm 1 \mathrm{~m} / \mathrm{s})$ with the corresponding $H_{S}$ and $T_{p}(1.7 \mathrm{~m}$ and $5.88 \mathrm{~s})$ as in section 4.1 or, on the other hand, to study the entire range $\left(V_{\text {in }}<V_{r}<V_{\text {out }}\right)$ with the conditioned $H_{S}$ and $T_{p}$ for each mean wind speed. These two approaches are performed with two random variables. In 
addition, to study the influence of waves the two sets are run also for still water, regular waves and irregular waves, Table 3 summarises the simulations.

The results -using again Von Mises stress at tower base- from all the simulations are captured in Figure 8 with the kriging model built with linear and matern 5/2 regression and correlation function correspondingly. One can see how expanding the range of a random variable increases the error and the number of sample points acquire more relevance, whereas restricting the variable to a smaller range increases accuracy. Also, the error for regular waves and still water is very small and decreases with increasing number of sample points in both cases, whereas the error with irregular waves decreases too if $V_{\text {in }}<V_{r}<V_{\text {out }}$, but it does not seem to be influenced by the number of sample points $V_{r}=[10,12]$.

\subsection{Wind turbulence}

Turbulence is the variation of the mean wind speed over time and space and it is introduced to the aero-elastic simulations through turbulence intensity $\left(\sigma_{1}\right)$ (IEC 2009). This parameter depends on the wind turbine class or the turbulence model and affects the standard deviation of the wind speed profile. For the NTM, it is: $\sigma_{1}=I_{r e f}\left(0.75 V_{h u b}+5.6\right)$. Proceeding the same way as in Table 3, a large number of simulations is carried with still water and different turbulence intensities (12,14 and 16\%) for different sets of sample points and two ranges of $V_{r}$

Figure 9 shows the RMSE of the Von Mises stress at tower base for each combination. Here, one can see the same pattern: with $V_{\text {in }}<V_{r}<V_{\text {out }}$ there is a decreasing RMSE with the number of sample points, whereas for $V_{r}=[10,12]$ this trend is less noticeable. However, the error is lower for all combinations. It is remarkable what an error the turbulent wind introduces to the model, although the difference between higher or lower turbulence intensities is negligible. 


\subsection{Number of seeds}

In this sub-section, the number of seeds used to obtain each sample point is studied. In this case, the simulations are carried out with a turbulent wind field as well as stochastic irregular waves. The range of the mean wind speed is chosen to be $V_{r}=[10,12]$. It is important to mention that this study implies high computational effort as the simulations required for each set of sample points are the product of the number of sample points and seeds, leading to a very large number of simulations. Due to computational limitations, only the set with 75 sample points is picked, and 2, 4, 6 and 8 seeds are used to obtain each sample point to construct the model. This methodology would be closer to what the code (IEC 2009) requires, which is six 10-minute simulations in case there is stochastic wind or waves. In this case, the average of the highest computed loads for different stochastic realisations shall be taken.

The results are shown in Figure 10a, where a nice decreasing trend over the number of seeds is observed. Two relevant ideas can be inferred from this figure, first is that even with just one seed for each sample point the RMSE is just about 2\%; and second, it seems that, in order to reduce the RSME to half, the number of sample points must be quadrupled. Also, it is interesting to see how the larger the number of seeds, the flatter the model, and the lesser the error and variability. This subsection is useful to validate the use of just one seed for the purpose of this study. Besides, it is remarkable that the response of the structure, in this case Von Mises stress, under stochastic loading can be approximated with less than $2 \%$ of RMSE when using an adequate kirging model. Summarising the results from the previous subsections, from an RMSE perspective, this methodology seems promising when estimating the outcome of a fully-coupled time-domain simulation, which includes inherent stochasticity in wind and waves. Here, one can also compare between the results for 1 seed and those from the previous sub-sections: looking at Figure 8 and Figure 9 one can also say that the error coming from stochastic wind and waves does not seem to be additive. The error with 75 
sample points with steady wind and irregular waves and still water and turbulent wind are 1.8 and $2.2 \%$ respectively, and up to $2.8 \%$ when using turbulent wind and irregular waves simultaneously.

\section{Reliability analysis based on DLCs}

In the final stage of computations, a reliability analysis is performed by using the kriging models to approximate any output from the fully-coupled simulations, with reference to the considered LSFs. In this case, more random variables are incorporated to the surrogate model, and the kriging models are included in the LSFs. For the reliability analysis, Monte Carlo simulations (10e6 simulations), FORM and SORM are computed. To apply FORM, the Matlab® toolbox FERUM (Der Kiureghian et al. 2006) is used. The considered LSFs are summarised in Table 4. The subscript krig indicates the kriging approximation/model of the variable, as a function of the 7 random variables considered.

The variables included in Table 4 are described in Table 5, which are implicit stochastic variables used to build the kriging models, e.g. $g_{2}=$ $g_{2}\left(D, \chi_{y^{\prime}} f_{y} E, \chi_{m}, t_{t}, t_{p l a t}, V_{w}, T_{p}, E I, L\right)$. The yield stress is considered as a random variable through the parameter $\chi_{y}$, and $\chi_{m}$ includes the model uncertainties.

The reliability techniques are applied to derive the reliability indexes $(\beta)$ for each of the LSFs and DLCs. The results showed that the LSFs related to buckling and plastic yielding are very far from failure; from where that a null probability of failure is computed using the three methods. To understand that, the critical moment is calculated for the mean values and it shows a difference of one order of magnitude with the highest bending moments at tower base levels. The reason for that is that the NREL 5MW monopile was created for code comparisons; it was not so much "designed", but rather "specified" based on industry trends and information from other reference turbines. In addition, these types of support structures, 
in general, are expected to fail more likely under fatigue limit states rather than ULS. These results agree with the overall conclusions on the literature of reliability of such structures (Muskulus and Schafhirt 2015). Also, the analysis of LSF $g_{4}$ showed that the gap between the tower and the blade tip is large enough even in the worst conditions. The DLC 6.2a is not considered for $g_{4}$ as it simulates parked/idling conditions with a fault on the yaw controller, in these cases the rotor would be stopped avoiding any interaction of blade/tower.

On the other hand, supporting the above-mentioned theory, the results showed a very low probability of failure for $g_{1}$, as seen in Table 6 . However, it is to be noted that $g_{1}$ is very conservative as these types of structures will unlikely fail by one element reaching yield: for a section to yield all the corresponding elements must yield first. Some of the $\beta$ 's are not given as they relate to too small probabilities of failure. For the case of $g_{5}$ and $g_{6}$ the limit is taken to show how the reliability indices evolve within a range between 8-9 $\mathrm{cm}$ and $0.5-0.6$ degrees, respectively.

Finally, the computational savings using the presented framework with the kriging approach against crude Monte Carlo simulations are shown averaged in Table 7. Although Monte Carlo simulations do not need to obtain the sample points, it needs to run the whole interface for each evaluation of the LSF. However, with the kriging method the main computational time is spent in obtaining the outputs of each sample point but the creation of kriging model and the evaluation of the LSF take only seconds. Another powerful advantage of the presented framework is that the cost of evaluating a new LSF is negligible, whereas crude MCS would require running the interface thousands of times again. It allows one to perform a comprehensive component-based reliability analysis with different LSFs at all levels with almost no extra computational cost.

As a subsequent part of the FORM analysis, a sensitivity analysis is performed to understand which variables play a more important role in defining the performance, as efforts 
to refine the uncertainty of these variables would have a greater impact on the reliability performance. From Table 7, it can be noticed that the DLC 1.6a is the most demanding and therefore it is picked for the sensitivity analysis. The results are shown in Table 8 for the considered LSFs. As it may be expected, the stochastic parameters corresponding to the uncertainty in the yield strength and in the Kriging model draw all the relevance and the variables included implicitly in the kriging model become residual. Hence efforts to improve the understanding about $\chi_{y}$ and $\chi_{m}$ would drastically increase the reliability of these type of structures. To have a clearer view of the influence of these variables the same analysis is carried out without $\chi_{y}$ and $\chi_{m}$ and the results are shown in Table 9. Of course, the probabilities of failure become smaller as part of the uncertainty is being deducted from the problem, but one can see the role of the other variables in a clearer way. It is interesting to see the influence of the thickness of the platform and the wave height in $g_{1}$. Moreover, the LSFs $g_{5}$ and $g_{6}$ show great correlation with the flexural rigidity $E I$ leaving the influence of $L$ as residual.

\section{Conclusions}

The objective of the present work is to demonstrate the application of an efficient responsebased approach to determine structural reliability of OWT support structures. Also, the most common LSFs in the literature relevant to different structural components are identified. Specific combinations of DLCs 1.3, 1.6a and 6.2a from IEC 61400-3 are picked as a proven set of the severest loading combinations by the literature. The NREL 5MW turbine is considered for the study modeled with a flexible foundation following the Apparent Fixity model. A dynamic interface between FAST and Abaqus ${ }^{\circledR}$ capable of performing efficient time-domain simulations is developed to obtain sample points to be used in the surrogate model. The influence of the turbulence, stochastic waves, and variable ranges are studied, 
along with an identification scheme for appropriate kriging models. The Kriging method is adopted here to develop the response surface for the desired outputs, such as the maximum Von Mises stress, bending moment at tower base level and blade-tower clearance. Six LSFs involving yield or local buckling to pile tip displacement and rotation are proposed. The probability of failure for each DLC combination and LSF is evaluated using Monte-Carlo simulations, FORM and SORM and a sensitivity analysis is performed. The present work is aimed as a demonstration tool for the application of the kriging approach to substitute the computationally expensive aero-elastic and FE time-domain simulations and efficiently approximate the responses of offshore wind turbine support structures. Also, a thorough analysis is performed on the influence of using different regression and correlation functions in the kriging model. The results agreed with recent literature showing very low or even zero probabilities of failure for most of the LSFs.

This framework considers uncertainties related to the soil-pile interaction through the flexural rigidity and the equivalent length, but it could be interesting to see the relationship between these parameters and actual parameters such as friction angle. In addition, as the accuracy of the methodology is analysed in an overall manner, further investigation is recommended to study the corresponding accuracy around the failure region, this may include advanced algorithms to improve the sampling technique.

\section{Acknowledgments}

This $\mathrm{PhD}$ research is funded by Lloyd's Register Group Services Ltd., Aberdeen. Sriramula's work within the Lloyd's Register Foundation Centre for Safety and Reliability Engineering at the University of Aberdeen. The Foundation helps to protect life and property by supporting engineeringrelated education, public engagement and the application of research.

\section{References}

Barker TB. 2005. Quality by experimental design. CRC Press.

Box GE, Hunter WG, Hunter JS. 1978. Statistics for experimenters. 
Bucher C, Bourgund U. 1990. A fast and efficient response surface approach for structural reliability problems. Struct Saf. 7(1):57-66.

Bush E, Manuel L. 2009. Foundation models for offshore wind turbines. ASME wind energy symposium, AIAAOrlando, Florida.

Carswell W, Arwade S, Myers A, Hajjar J. 2014a. Reliability analysis of monopile offshore wind turbine support structures. Safety, Reliability, Risk and Life-Cycle Performance of Structures and Infrastructures.223.

Carswell W, Arwade SR, DeGroot DJ, Lackner MA. 2014b. Soil-structure reliability of offshore wind turbine monopile foundations. Wind Energy.

Couckuyt I, Dhaene T, Demeester P. 2014. ooDACE toolbox: A flexible object-oriented kriging implementation. The Journal of Machine Learning Research. 15(1):3183-6.

Der Kiureghian A, Haukaas T, Fujimura K. 2006. Structural reliability software at the university of california, berkeley. Struct Saf. 28(1):44-67.

DNV. 2010. Fatigue design of offshore steel structures. Recommended practice DNV-RPC203. Det Norske Veritas.

Fischer T, De Vries W, Schmidt B. 2010. UpWind design basis (WP4: Offshore foundations and support structures). Project UpWind.

Gaspar B, Teixeira A, Soares CG. 2014. Assessment of the efficiency of kriging surrogate models for structural reliability analysis. Prob Eng Mech. 37:24-34.

Hohenbichler M, Gollwitzer S, Kruse W, Rackwitz R. 1987. New light on first-and secondorder reliability methods. Struct Saf. 4(4):267-84.

IEC. 2009. IEC 61400-3: Wind Turbines-Part 3: Design requirements for offshore wind turbines. International Electrotechnical Commission, Geneva.

IEC. 2005. IEC 61400-1: Wind Turbines-Part 1: Design requirements. International Electrotechnical Commission, Geneva.

Jha A, Dolan D, Gur T, Soyoz S, Alpdogan C. 2009. Comparison of API \& IEC standards for offshore wind turbine applications in the US atlantic ocean: Phase II. Contract. 303:275-3000.

Jonkman BJ. 2009. TurbSim user's guide: Version 1.50. National Renewable Energy Laboratory Golden, CO, USA.

Jonkman JM, Buhl MLJ. 2005. FAST user's guide - Technical report NREL/EL-500-38230 national renewable energy laboratory. Colorado, USA. Report nr 144 pp. 
Jonkman JM, Butterfield S, Musial W, Scott G. 2009. Definition of a 5-MW reference wind turbine for offshore system development. Colorado, USA: National Renewable Energy Laboratory. Report nr NREL/TP-500-38060.

Kaymaz I. 2005. Application of kriging method to structural reliability problems. Struct Saf. 27(2):133-51.

Kim DH, Lee SG. 2015. Reliability analysis of offshore wind turbine support structures under extreme ocean environmental loads. Renewable Energy. 79:161-6.

Lataniotis C, Marelli S, Sudret B. 2015. UQLAB user Manual-Kriging (gaussian process modelling), report UQLab-V0.9-105, chair of risk, safety \& uncertainty quantification. ETH, Zurich.

Morató Casademunt A, Sriramula S, Krishnan N. Sept 2016. Reliability analysis of offshore wind turbine support structures using kriging models. Safety and reliability of complex engineered systems, ESREL 2016Glasgow, UK.

Morató Casademunt A, Sriramula S, Krishnan N. Sept 2015. A computational framework for the reliability of offshore wind turbine support structures. Safety and reliability of complex engineered systems, ESREL 2015Zurich, Switzerland. 4181-4187 p.

Morató A, Sriramula S, Krishnan N, Nichols J. 2017. Ultimate loads and response analysis of a monopile supported offshore wind turbine using fully coupled simulation. Renewable Energy. 101:126-43.

Muskulus M, Schafhirt S. 2015. Reliability-based design of wind turbine support structures. Symposium on Reliability of Engineering System. SRES'2015. Hangzhou, China. 111.

NORSOK. 2004. Design of steel structures. standard N-004. Lysaker: Standards Norway.

Norton E, Quarton D. 2003. Recommendations for design of offshore wind tubines (RECOFF). D3 Deliverable-Collated Sensitivity Studies, Document No. 2762.

Rasmussen CE. 2006. Gaussian processes for machine learning.

Sacks J, Schiller SB, Welch WJ. 1989. Designs for computer experiments. Technometrics. 31(1):41-7.

Santner TJ, Williams BJ, Notz WI. 2013. The design and analysis of computer experiments. Springer Science \& Business Media.

Shi X, Palos Teixeira Â, Zhang J, Guedes Soares C. 2015. Kriging response surface reliability analysis of a ship-stiffened plate with initial imperfections. Structure and Infrastructure Engineering. 11(11):1450-65.

Sørensen JD, Toft HS. 2010. Probabilistic design of wind turbines. Energies. 3(2):241-57. 
Sorensen JD, Tarp-Johansen N. 2005. Reliability-based optimization and optimal reliability level of offshore wind turbines. International Journal of Offshore and Polar Engineering. 15(02).

Wang GG. 2003. Adaptive response surface method using inherited latin hypercube design points. Transactions-American Society of Mechanical Engineers Journal of Mechanical Design. 125(2):210-20.

Wei K, Arwade SR, Myers AT. 2014. Incremental wind-wave analysis of the structural capacity of offshore wind turbine support structures under extreme loading. Eng Struct. 79:58-69.

Yang H, Zhu Y, Lu Q, Zhang J. 2015. Dynamic reliability based design optimization of the tripod sub-structure of offshore wind turbines. Renewable Energy. 78:16-25.

Zhang L, Lu Z, Wang P. 2015. Efficient structural reliability analysis method based on advanced kriging model. Appl Math Model. 39(2):781-93. 


\section{Figures}
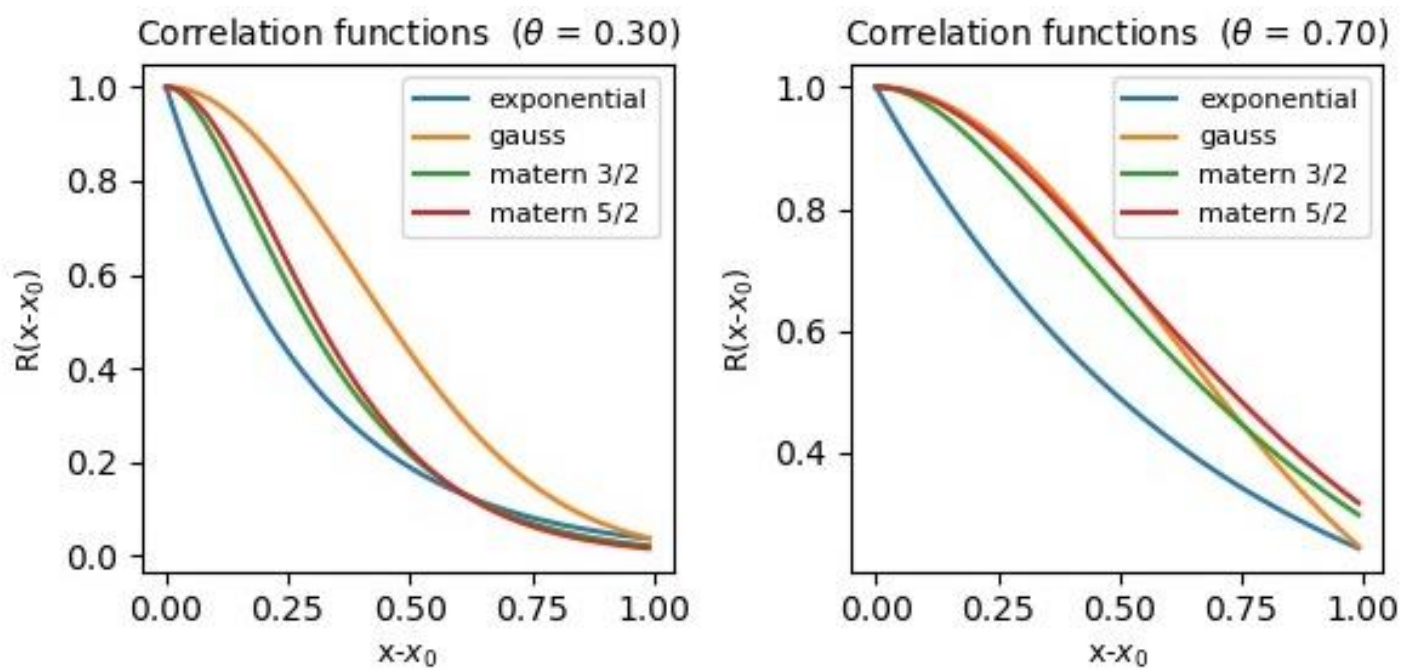

Figure 1 - Correlation functions and the effect of the hyperparameters

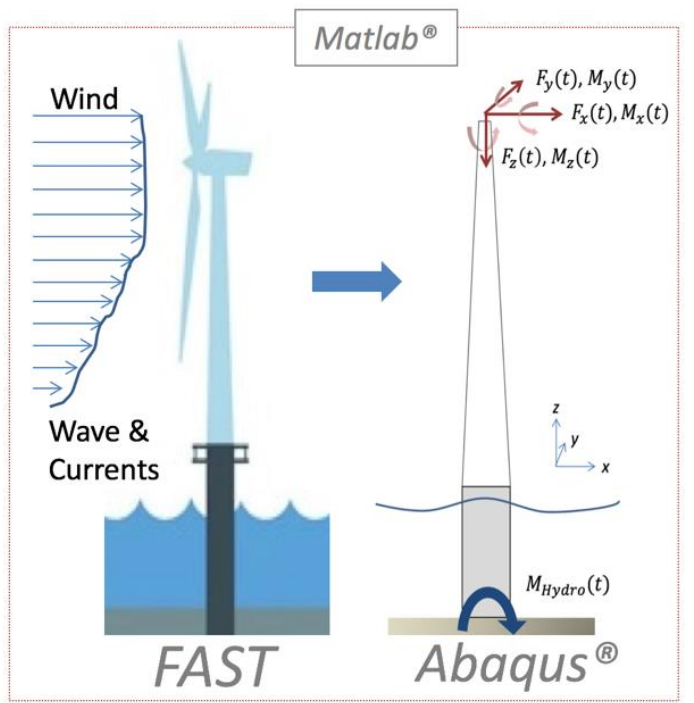

Figure 2 - Scheme of the interface between FAST and Abaqus ${ }^{\circledR}$ 


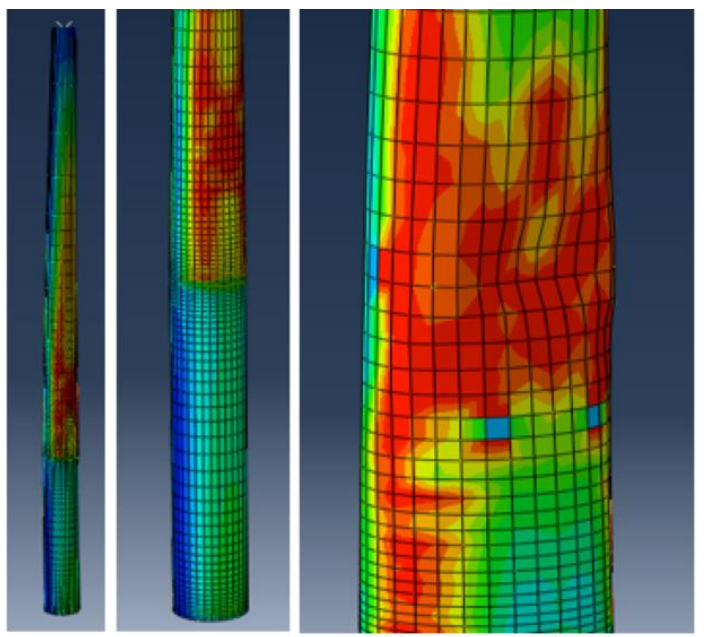

a)

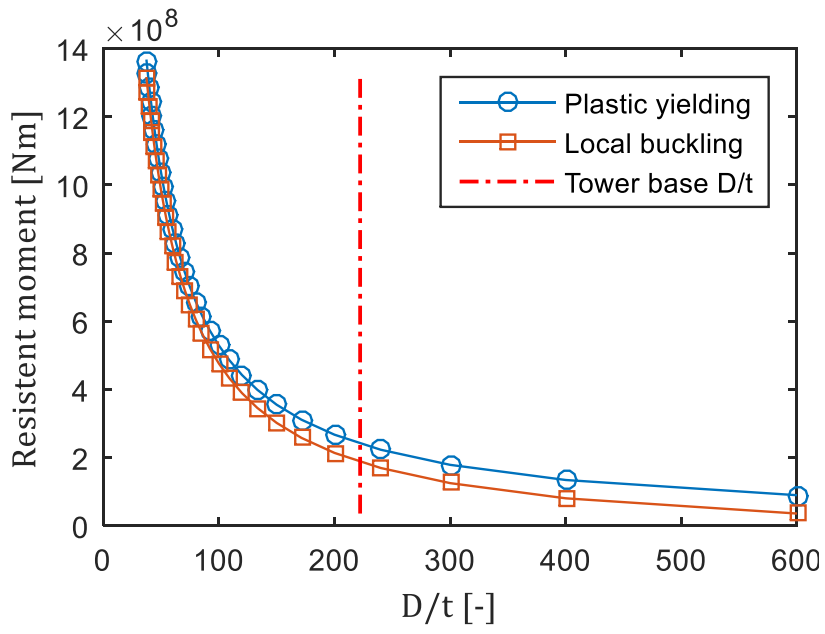

b)

Figure 3 - a) Example of local buckling. b) Section plastic moment and simplified model for the buckling resistance as a function of $\mathrm{D} / \mathrm{t}$

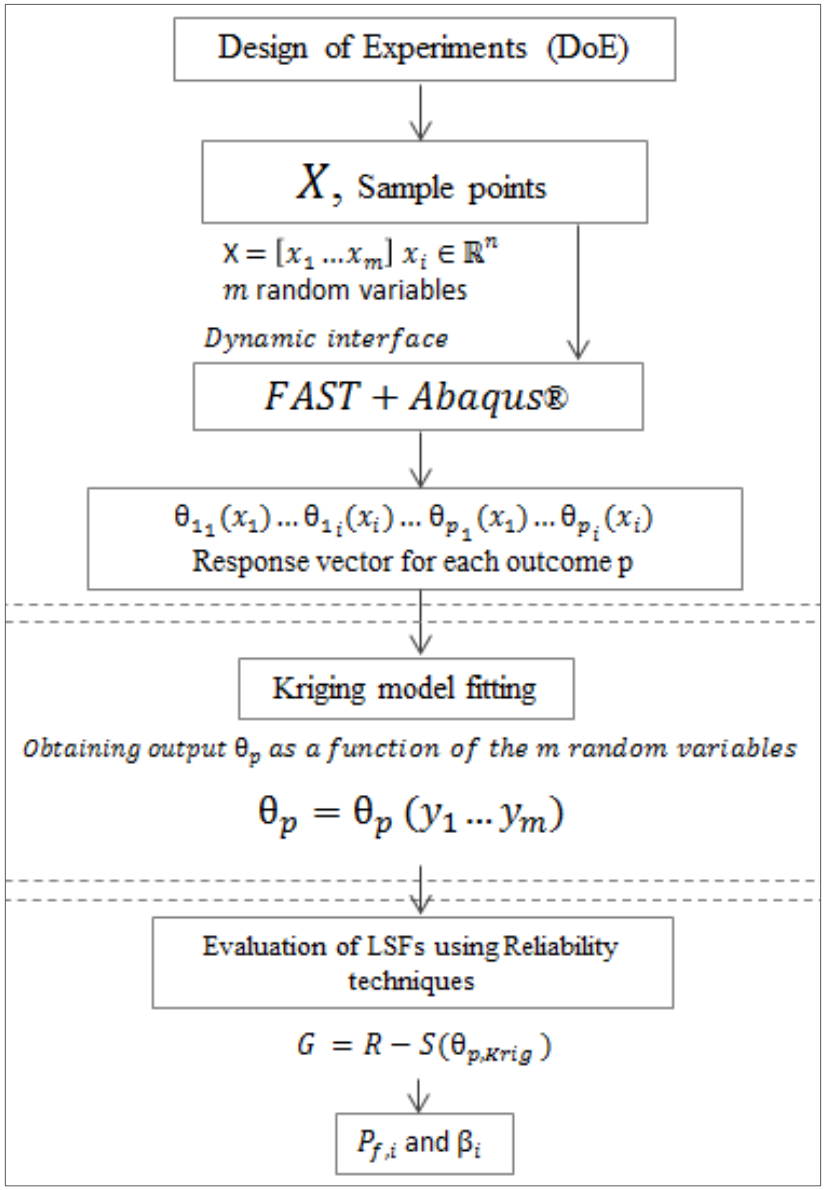

Figure 4 - Flow chart of the procedure to create the kriging model and analyse the reliability 


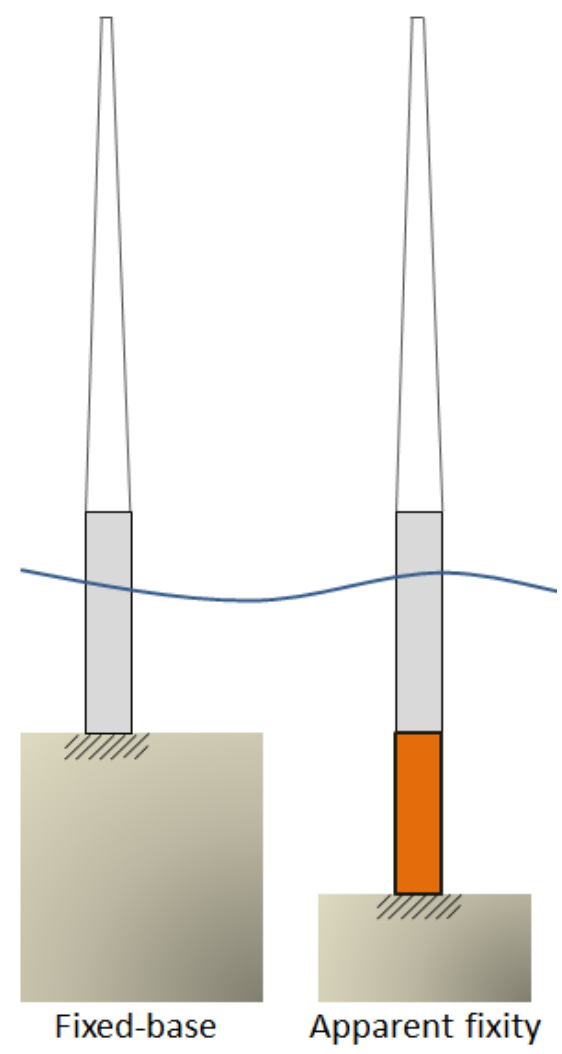

Figure 5 - Foundation models 


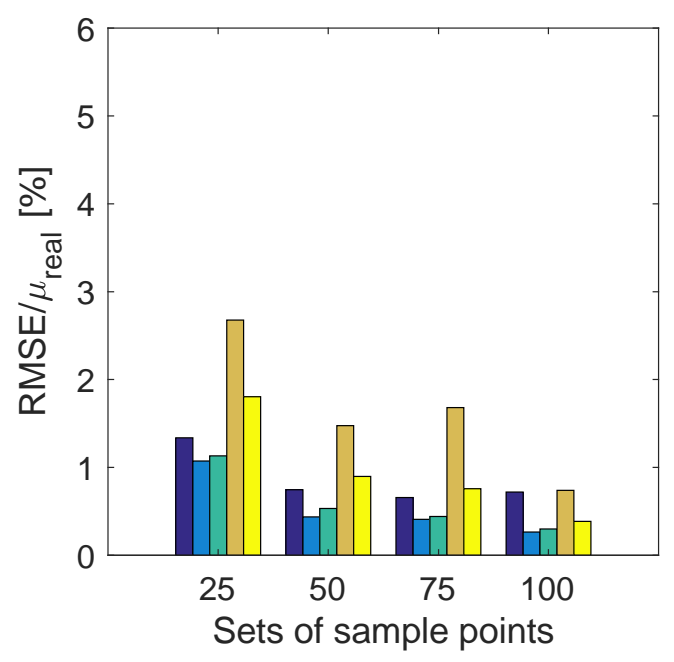

a) Steady wind and still water

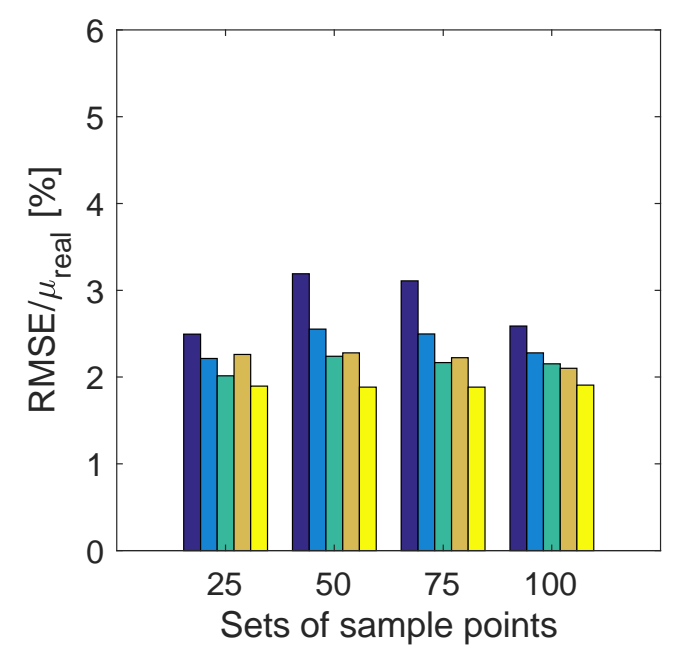

c) Turbulent wind and regular waves

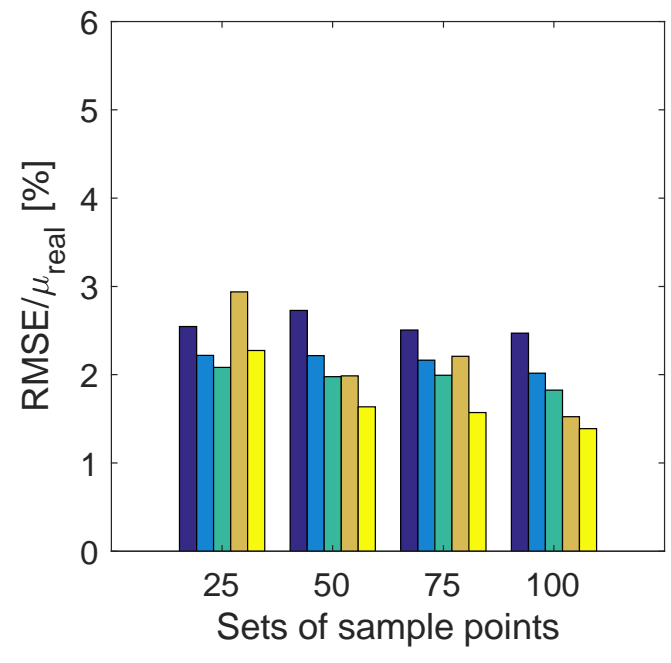

b) Steady wind and Irregular waves

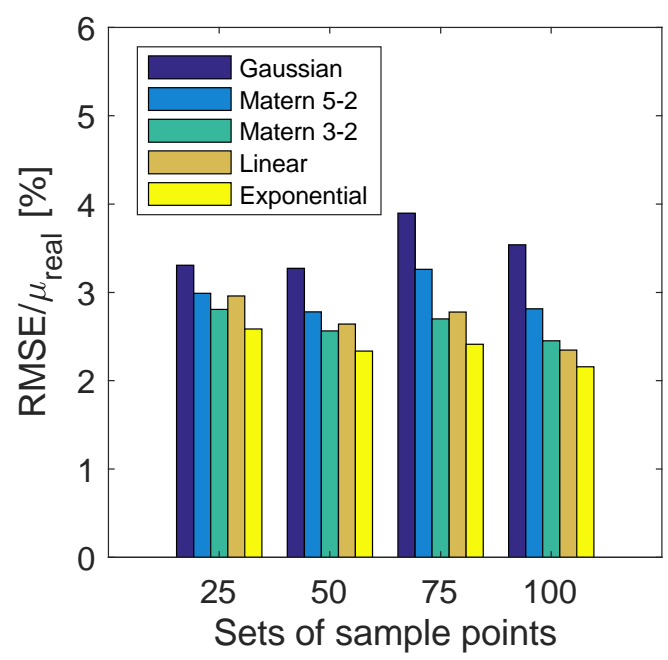

d) Turbulent wind and irregular waves

Figure 6 - Evolution of the RMSE using different correlation functions and number of sample points as well as inherent variability introduced by wind turbulence and random wave profiles. 


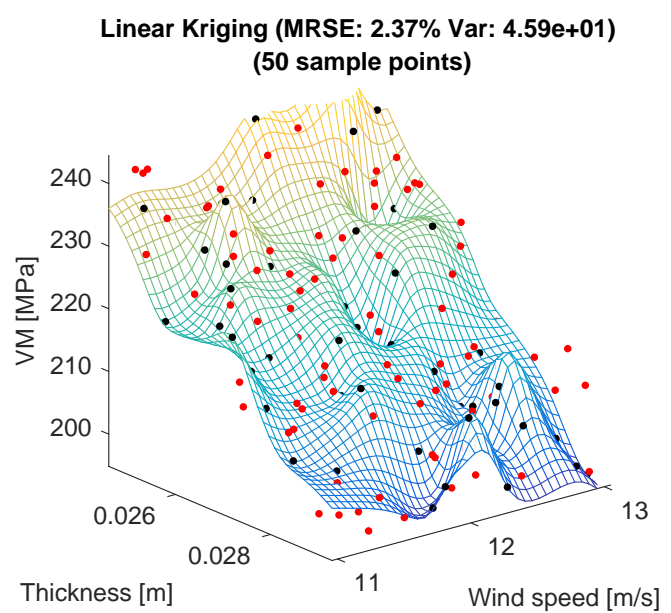

a)

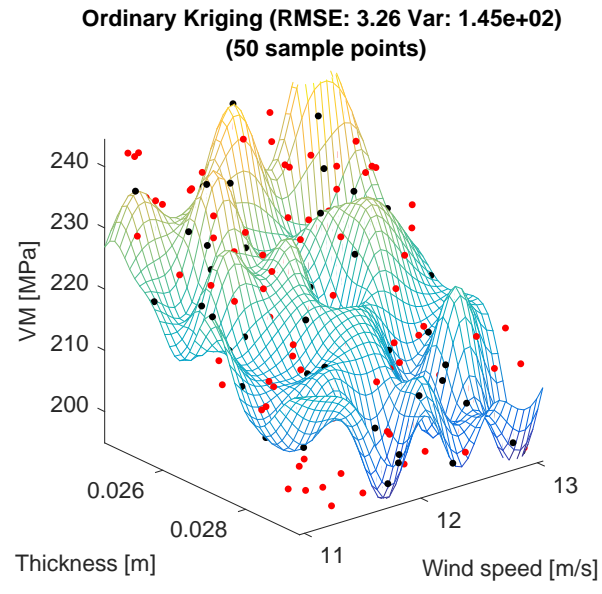

b)

Figure 7 - Kriging models using linear regression function and Matern5/2 correlation function (a) and ordinary kriging with exponential correlation function (b)
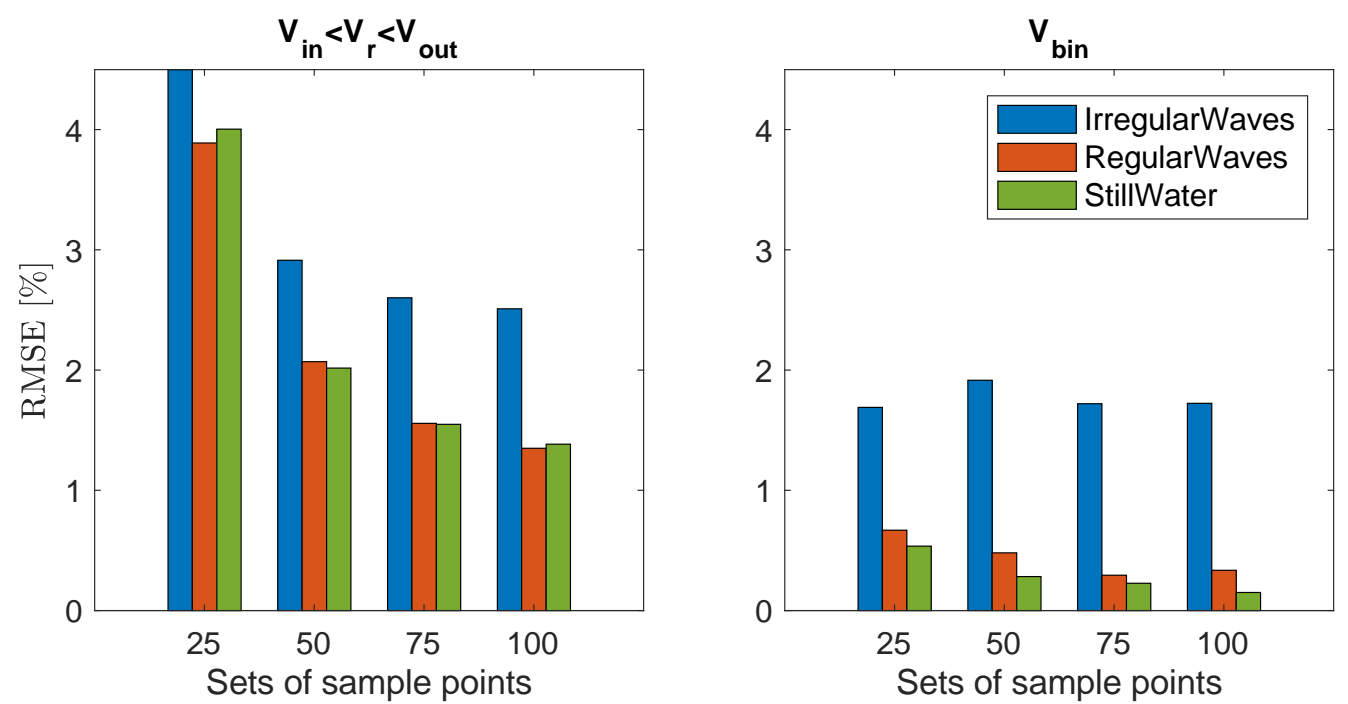

Figure 8 - Influence of regular and stochastic irregular waves 

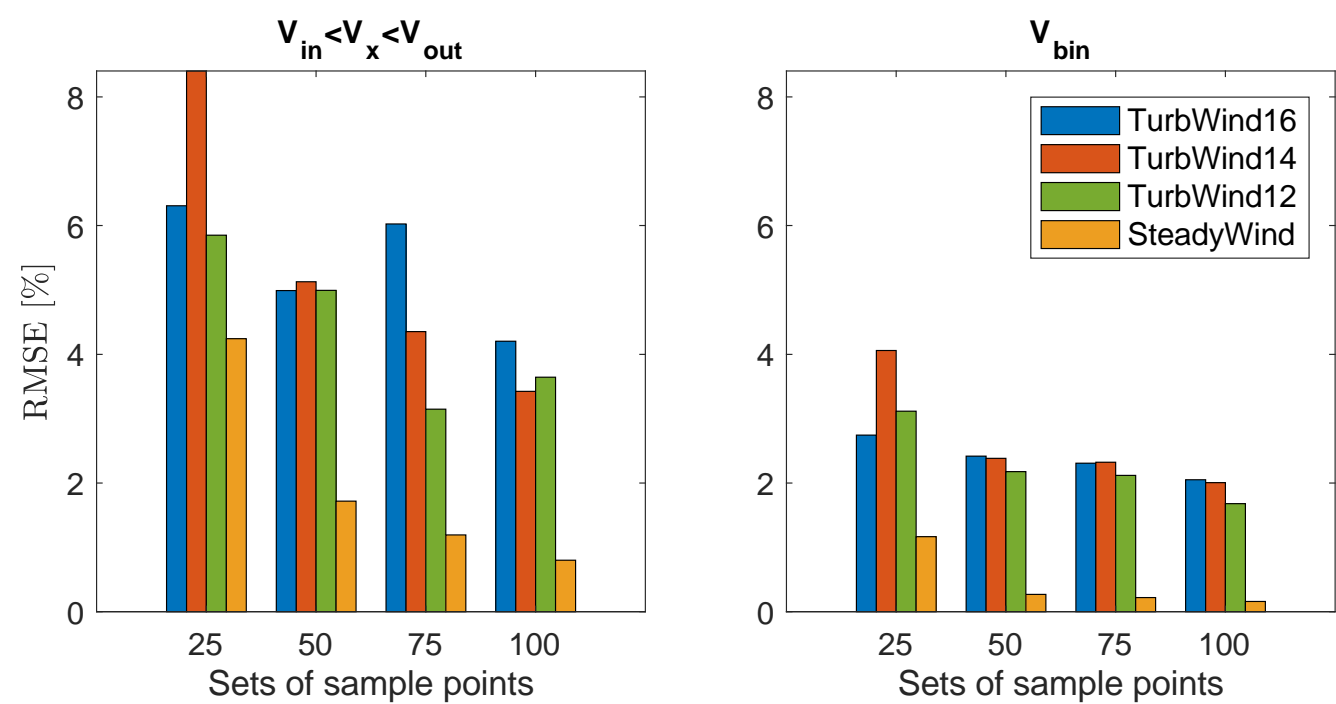

Figure 9 - Influence of wind turbulence and the number of sample points
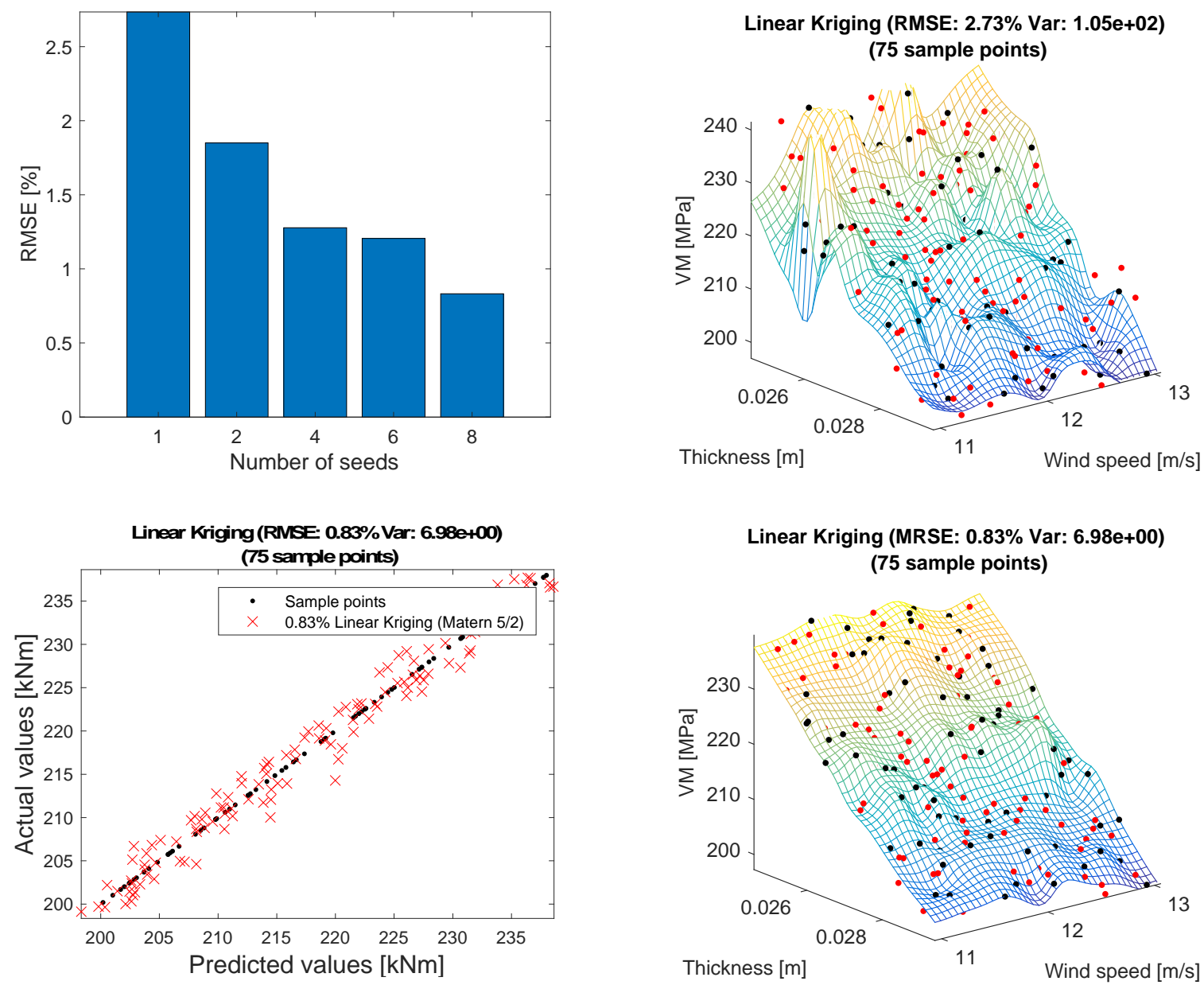

Figure 10 - Influence of number of seeds using a set of 75 sample points to build the kriging model 


\section{Tables}

Table 1 - Apparent fixity model parameters for DLCs 1.3, 1.6a and 6.2a

\begin{tabular}{ccc}
\hline DLC & $\begin{array}{c}\text { Flexural } \\
\text { rigidity, EI } \\
{[\mathrm{N} \cdot \mathrm{m} 2]}\end{array}$ & $\begin{array}{c}\text { Equivalent } \\
\text { length, } \mathrm{L} \\
{[\mathrm{m}]}\end{array}$ \\
\hline 1.3 & $1.050 \mathrm{e} 12$ & 16.320 \\
$1.6 \mathrm{a}$ & $1.037 \mathrm{e} 12$ & 16.450 \\
\hline $6.2 \mathrm{a}$ & $1.039 \mathrm{e} 12$ & 16.381 \\
\hline
\end{tabular}

Table 2 - Summary of DLCs

\begin{tabular}{|c|c|c|c|c|c|c|c|c|}
\hline \multirow[b]{2}{*}{ DLC } & \multicolumn{2}{|c|}{ Wind } & \multicolumn{2}{|c|}{ Waves } & \multicolumn{2}{|c|}{ Current } & \multirow{2}{*}{$\begin{array}{c}\text { Yaw } \\
\text { angle }\end{array}$} & \multirow{2}{*}{$\begin{array}{c}\text { Wind/wave } \\
\text { angle }\end{array}$} \\
\hline & Model & $\begin{array}{l}\text { Speed } \\
{[\mathrm{m} / \mathrm{s}]}\end{array}$ & Model & $H_{S}[\mathrm{~m}] \mid T_{p}[\mathrm{~s}]$ & Model & $\begin{array}{l}\text { Speed } \\
{[\mathrm{m} / \mathrm{s}]}\end{array}$ & & \\
\hline \multicolumn{9}{|c|}{ Power production } \\
\hline 1.3 & ETM & 14 & NSS & \begin{tabular}{l|l}
1.91 & 6.07
\end{tabular} & NCM & 0.6 & $8^{\circ}$ & $0^{\circ}$ \\
\hline $1.6 \mathrm{a}$ & NTM & 12 & SSS & $8.07 \mid 11.3$ & NCM & 0.6 & $8^{\circ}$ & $0^{\circ}$ \\
\hline \multicolumn{9}{|c|}{ Parked / idling } \\
\hline $6.2 \mathrm{a}$ & EWM & 40.375 & ESS & 11.3 & $\mathrm{ECM}$ & 1.2 & $90^{\circ}$ & $30^{\circ}$ \\
\hline
\end{tabular}

Table 3 - Simulations sets for sensitivity analysis

\begin{tabular}{|c|c|c|c|}
\hline $\begin{array}{l}\text { Sets of } \\
\text { simulations }\end{array}$ & Conditions & $\begin{array}{l}\text { Random } \\
\text { variables/range }\end{array}$ & $\begin{array}{l}\text { Experimental } \\
\text { points }\end{array}$ \\
\hline $1^{\text {st }}$ Set & Steady wind & $\begin{array}{l}V_{r}=[10,12] \\
t_{\text {tower }}=[-3 \sigma,+3 \sigma]\end{array}$ & $25,50,75,100$ \\
\hline $2^{\text {nd }}$ Set & Still water & 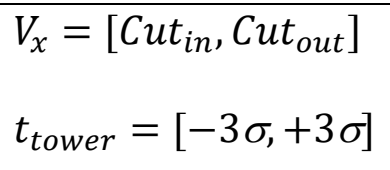 & $25,50,75,100$ \\
\hline $3^{\text {rd }}$ Set & Steady wind & $\begin{array}{l}V_{r}=[10,12] \\
t_{\text {tower }}=[-3 \sigma,+3 \sigma]\end{array}$ & $25,50,75,100$ \\
\hline $4^{\text {th }}$ Set & Regular waves & 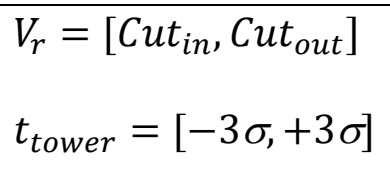 & $25,50,75,100$ \\
\hline $5^{\text {th }}$ Set & $\begin{array}{l}\text { Steady wind } \\
\text { Irregular }\end{array}$ & $\begin{array}{l}V_{r}=[10,12] \\
t_{\text {tower }}=[-3 \sigma,+3 \sigma]\end{array}$ & $25,50,75,100$ \\
\hline $6^{\text {th }}$ Set & waves & $V_{r}=\left[\right.$ Cut $_{\text {in }}$, Cut $\left.t_{\text {out }}\right]$ & $25,50,75,100$ \\
\hline
\end{tabular}




$$
t_{\text {tower }}=[-3 \sigma,+3 \sigma]
$$

Table 4 - List of Limit State Functions

\begin{tabular}{|c|c|c|}
\hline & Description & LSF \\
\hline & Von Mises stress & \\
\hline$g_{1}$ & reaching yield & $G=\chi_{y} f_{y}-X_{m} \sigma_{V M, \text { Krig }}$ \\
\hline$g_{2}$ & $\begin{array}{l}\text { Simplified tower } \\
\text { buckling model }\end{array}$ & $G=\frac{1}{6}\left(1-0.84 \frac{D}{t_{t}} \frac{\chi_{y} f_{y}}{E}\right)\left(D^{3}-\left(D-2 t_{t}\right)^{3}\right) \chi_{y} f_{y}-\chi_{m} M_{\text {Krig }}$ \\
\hline$g_{3}$ & Plastic yielding & $G=\frac{1}{6}\left(D^{3}-\left(D-2 t_{t}\right)^{3}\right) \chi_{y} f_{y}-\chi_{m} M_{K r i g}$ \\
\hline & Blade - tower & \\
\hline$g_{4}$ & clearance & $G=\chi_{m}$ Clear $_{\text {Krig }}$ \\
\hline$g_{5}$ & $\begin{array}{c}\text { Pile top } \\
\text { displacement }\end{array}$ & $G=\omega_{\text {lim }}-\chi_{m} M u d D x_{\text {Krig }}$ \\
\hline$g_{6}$ & Pile top rotation & $G=\alpha_{l i m}-\chi_{m} M u d D x_{K r i g}$ \\
\hline
\end{tabular}

Table 5 - Stochastic and deterministic variables used in the LSFs and the kriging models (Sørensen and Toft 2010; Sorensen and Tarp-Johansen 2005)

\begin{tabular}{|c|c|c|c|c|}
\hline & Description & $\begin{array}{c}\text { Distribution } \\
\text { type }\end{array}$ & $\begin{array}{l}\text { Expected } \\
\text { value }\end{array}$ & $\mathrm{CoV}$ \\
\hline \multicolumn{5}{|c|}{ Random variables implicit in kriging models } \\
\hline$t_{t}$ & Thickness at tower base level & Normal & $0.027 \mathrm{~m}$ & 0.03 \\
\hline$t_{\text {plat }}$ & Platform thickness & Normal & $0.06 \mathrm{~m}$ & 0.03 \\
\hline$V_{w}$ & Mean wind speed & Normal & Table 2 & 0.05 \\
\hline$H_{s}$ & Significant wave height & Normal & Table 2 & 0.05 \\
\hline$T_{p}$ & Peak spectral period & Normal & Table 2 & 0.05 \\
\hline$E I$ & $\begin{array}{c}\text { Flexural rigidity of the } \\
\text { foundation }\end{array}$ & Normal & Table 1 & 0.05 \\
\hline$L$ & $\begin{array}{l}\text { Equivalent length of the } \\
\text { foundation }\end{array}$ & Normal & Table 1 & 0.05 \\
\hline \multicolumn{5}{|c|}{ Random parameters in LSFs } \\
\hline$\chi_{y}$ & Yield Stress coefficient & Lognormal & 1 & 0.05 \\
\hline$\chi_{m}$ & Kriging model coefficient & Normal & 1 & 0.2 \\
\hline Deter & ministic variables & & & \\
\hline
\end{tabular}




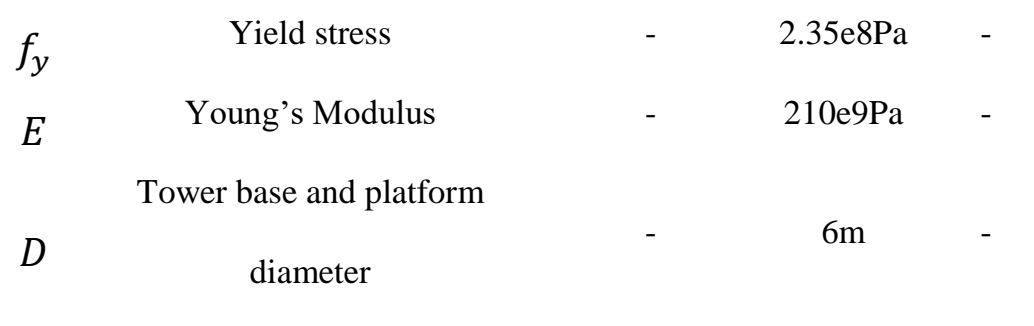

Table 6 - Reliability indices $(\boldsymbol{\beta})$ for each DLC and LSF using MCS, FORM and SORM

\begin{tabular}{|c|c|c|c|c|c|c|}
\hline \multirow[b]{2}{*}{$\begin{array}{c}\text { DL } \\
\mathrm{C}\end{array}$} & \multirow{2}{*}{$\begin{array}{c}\text { Reliability } \\
\text { method }\end{array}$} & \multirow[b]{2}{*}{$g_{1}$} & \multicolumn{2}{|c|}{$g_{5}$} & \multicolumn{2}{|c|}{$g_{6}$} \\
\hline & & & $\omega_{\text {lim }}=8 \mathrm{~cm}$ & $\omega_{\text {lim }}=9 \mathrm{~cm}$ & $\alpha_{l i m}=0.4^{\circ}$ & $\begin{array}{l}\alpha_{\text {lim }} \\
=0.5^{\circ}\end{array}$ \\
\hline \multirow{3}{*}{1.3} & MCS & 3.881 & - & - & - & - \\
\hline & FORM & 4.547 & 9.856 & 11.89 & 8.701 & 10.567 \\
\hline & SORM & - & - & - & - & - \\
\hline \multirow{3}{*}{$1.6 \mathrm{a}$} & MCS & 4.224 & 3.956 & 4.935 & 2.284 & 4.038 \\
\hline & FORM & 4.278 & 3.953 & 5.010 & 2.284 & 4.043 \\
\hline & SORM & 4.286 & 3.963 & 5.023 & 2.278 & 4.031 \\
\hline \multirow{3}{*}{$6.2 \mathrm{a}$} & MCS & 4.133 & 4.175 & 5.069 & 2.880 & 4.526 \\
\hline & FORM & 4.312 & 4.225 & 5.216 & 2.970 & 4.567 \\
\hline & SORM & 4.140 & 4.177 & 5.175 & 3.246 & 4.453 \\
\hline
\end{tabular}

Table 7 - Comparison of computational times for 1 LSF

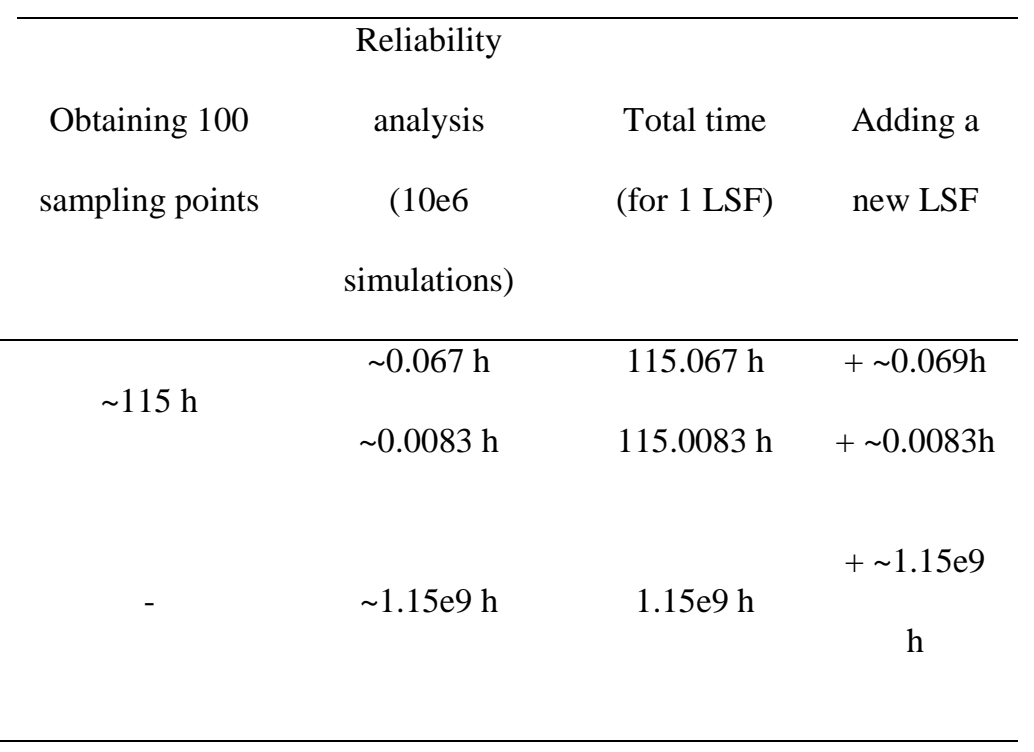

Table 8 - Sensitivity analysis for DLC 1.6a

\begin{tabular}{ccc}
\hline \multirow{2}{*}{$g_{1}$} & $g_{5}$ & $g_{6}$ \\
\cline { 2 - 3 } & $\omega_{\text {lim }}=8 \mathrm{~cm}$ & $\alpha_{\text {lim }}=0.5^{\circ}$ \\
\hline
\end{tabular}




\begin{tabular}{cccc}
\hline$t_{t}$ & - & $-0,053$ & -0.003 \\
$t_{\text {plat }}$ & $-0,030$ & 0,064 & 0.025 \\
$V_{w}$ & - & - & -0.001 \\
\hline$H_{s}$ & 0,057 & 0,176 & 0.167 \\
$T_{p}$ & - & - & - \\
$E I$ & - & $-0,167$ & -0.099 \\
\hline$L$ & - & - & - \\
$\chi_{y}$ & $-0,406$ & - & - \\
$\chi_{m}$ & 0,912 & 0,967 & 0.981 \\
\hline
\end{tabular}

Table 9 - Sensitivity analysis for DLC 1.6 a without the random parameters $\chi_{m}$ and $\chi_{y}$

\begin{tabular}{cccc}
\hline & $g_{1}$ & $g_{5}$ & $g_{6}$ \\
\cline { 3 - 4 } & - & 0.022 & -0.154 \\
\hline$t_{t}$ & - & $-0,439$ & 0.194 \\
$t_{\text {plat }}$ & 0,621 & - & -0.002 \\
$V_{w}$ & - & & 0.059 \\
\hline$H_{S}$ & $-0,784$ & 0,039 & - \\
$T_{p}$ & - & - & -0.967 \\
$E I$ & - & $-0,897$ & - \\
\hline$L$ & - & - &
\end{tabular}

